\title{
Research on Parameter Optimization Based on Genetic Algorithm to Suppress Transient Overvoltage of LCC-HVDC System
}

\author{
Qihui LIU ${ }^{1}$ and Yong WU \\ School of Electric and Electronic Engineering, North China Electric Power University, \\ Beijing, China
}

\begin{abstract}
Since the 1980s, the direct current (DC) transmission technology has developed rapidly in China. However, with the large-scale application of DC transmission technology, new problems are brought to the power system. Commutation failure of the DC system will cause overvoltage in the alternating current (AC) power network, which will cause great harm to the weak AC system with wind power access. Therefore, it is urgent to carry out research on overvoltage suppression at the sending end of AC system. This paper reveals the formation mechanism of transient overvoltage and confirms that the control parameters of DC system influence the voltage at the sending end directly. And the appropriate control parameters were selected to suppress the overvoltage by the parameter optimization based on genetic algorithm (GA).
\end{abstract}

Keywords. Direct current transmission technology, genetic algorithm, AC system

\section{Introduction}

Due to China's special energy structure, the energy is mainly distributed in the three north regions (Northwest, Northeast, North China) [1], while the load is mostly distributed in Central China and Eastern China, which makes the new energy power generation represented by wind power and high voltage direct current (HVDC)transmission have developed on a large scale in recent years. However, the large-scale wind power sent through the DC transmission system also brings new problems to the power system. The connection of DC system will make the transient voltage stability problem further complicated.

Relevant scholars at home and abroad have carried out related research on suppressing transient overvoltage and commutation failure, and formed some research results. A second-order differential control strategy is proposed to compensate the power instruction delay for the wind farm, which can effectively suppress the overvoltage at the sending end of the AC system [2]. It can be concluded that the rapid change of DC current results in the rapid change of reactive power absorbed by the rectifier, which mainly influences voltage at the sending end of the AC system [3]. In

${ }^{1}$ Corresponding Author, Qihui LIU, School of Electric and Electronic Engineering, North China Electric Power University, Beijing, China; Email: 1339133389@qq.com. 
Ref. [4], it is analyzed that static synchronous compensator (STATCOM) and static var compensator (SVC) significantly increase the stability level of the system voltage and suppress transient overvoltage, and STATCOM plays a more important role. In Ref. [5], a coordinated control research of stability control system and pole control system under DC system block is proposed, which also considers transient overvoltage at the sending end of the AC system. In Ref. [6], a high-speed commutation failure inhibition strategy is proposed, which can suppress overvoltage indirectly. Parameter optimization is proposed for current controller in HVDC control system, which can improve steadystate performance of HVDC system [7]. A parameter optimization method is proposed for commutation failure prevention in line commutation converter (LCC)-HVDC system, which improves the immunity of commutation failure [8]. The parameters of fan and DC system are coordinated and optimized to jointly suppress transient overvoltage in Ref. [9].

This paper firstly reveals the formation mechanism of transient overvoltage and confirms that the control parameters of DC system influence the voltage at the seeding end directly. Then the appropriate control parameters were selected to suppress the overvoltage by the parameter optimization based on GA. Finally, the effectiveness of the proposed parameter optimization method is verified in PSCAD/EMTDC.

\section{Mechanism of Suppressing the Overvoltage at the Sending End}

Reactive power exchange between the rectifier station and the AC power network is shown in figure 1 , where $Q_{W}$ is the reactive power output of the AC power network, $Q_{F}$ is the reactive power compensated by the reactive power compensation device and $Q_{d c}$ is the reactive power absorbed by the rectifier station.

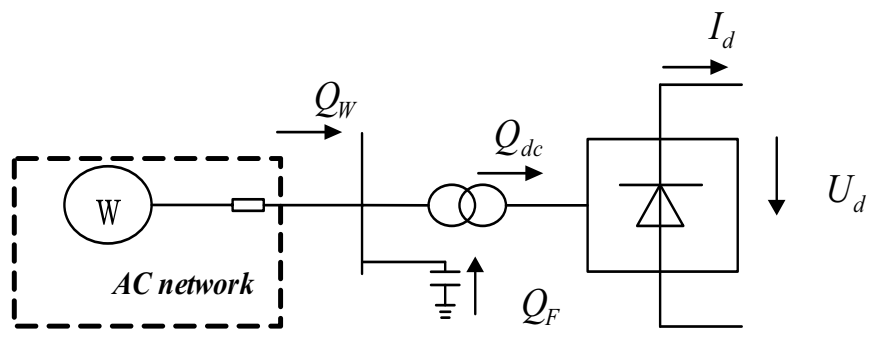

Figure 1. Schematic diagram of a HVDC transmission system.

When the system is in steady state,

$$
Q_{W}+Q_{F}=Q_{d c}
$$

In the dynamic process of the system,

$$
\begin{gathered}
Q_{c h}=Q_{W}+Q_{F}-Q_{d c} \\
\Delta U_{a c}=Q_{c h} / S_{d}
\end{gathered}
$$


where: $Q_{c h}$ is unbalanced reactive power of dynamic process, $\Delta U_{a c}$ is transient voltage change rate of the converter bus and $S_{d}$ is short circuit capacity of the AC system.

Based on (3), the strength of the AC system generally does not change in the dynamic process, so the voltage at the sending-end bus is mainly influenced by the unbalanced reactive power, which is related to $Q_{F}, Q_{W}$ and $Q_{d c}$ based on (2). In this paper, $Q_{d c}$ is main factor considered to change the voltage at the sending end.

\subsection{Mathematical Model of HVDC Transmission System}

In order to study the formation mechanism of overvoltage in HVDC transmission system, it is necessary to have a brief understanding of the mathematical model of HVDC transmission system. Figure 2 shows the equivalent circuit of the HVDC transmission system composed of two six-pulse converters in series:

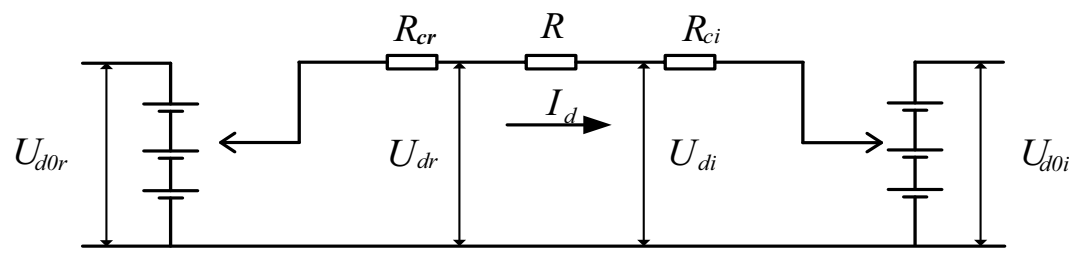

Figure 2. Equivalent circuit of HVDC transmission system.

In figure $2, U_{d 0 r}$ and $U_{d 0 i}$ are the ideal no-load DC voltage at the rectifying side and the inverting side respectively. $R_{c r}$ and $R_{c i}$ are equivalent commutation resistances at the rectifying side and inverting side respectively. $U_{d r}$ and $U_{d i}$ are the DC voltage output at the rectifying side and the inverting side respectively.

According to the working principle of the converter, the DC voltage of the rectifying side can be expressed as:

$$
V_{d r}=V_{d 0 r} \cos \alpha-I_{d} R_{c r}
$$

DC voltage of inverting side can be expressed as:

$$
V_{d i}=V_{d 0 i} \cos \beta+I_{d} R_{c i}
$$

Or

$$
V_{d i}=V_{d 0 i} \cos \gamma-I_{d} R_{c i}
$$

Then the DC current can be expressed as:

$$
I_{d}=\frac{V_{d r}-V_{d i}}{R}
$$

Or 


$$
I_{d}=\frac{\left(V_{d 0 r} \cos \alpha-V_{d 0 i} \cos \gamma\right)}{R_{c r}+R+R_{c i}}
$$

where $\alpha$ and $\beta$ are the leading trigger Angle of the rectifier and the hysteresis trigger Angle of the inverter respectively, and $\gamma$ is the turn-off Angle of the inverter.

Power absorbed by rectifier can be expressed as:

$$
\left\{\begin{array}{l}
P_{r}=U_{d r} I_{d} \\
Q_{r}=P_{r} \tan \varphi_{r}
\end{array}\right.
$$

where the active power and reactive power absorbed by the rectifier and the power factor angle of the rectifier are as follows:

$$
\varphi_{r}=\arctan \left[\frac{(\pi / 180) \mu-\sin \mu \cos (2 \alpha+\mu)}{\sin \mu \sin (2 \alpha+\mu)}\right]
$$

where $\mu$ is the commutation angle of the rectifier.

\subsection{Formation Mechanism of Transient Overvoltage}

When commutation failure occurs at the inverting side, the voltage at the sending end generally decreases first and then increases. The specific formation process can be divided into the following three stages:

(1) DC current increase stage: The commutation failure happens at the inverting side. At the same time, the DC voltage at the inverting side decreases rapidly to 0 . Based on (7), the DC current will rise accordingly, thus the reactive power absorbed by the rectifier will increase correspondingly, resulting in low voltage at the sending end.

(2) DC current reduction stage: Based on (8), due to the regulation of the current control amplifier (CCA) and the low-voltage current limiting link (VDCOL), the DC current will start to decrease with the increase of the trigger angle, even may drop to 0 , and the reactive power consumed by the rectifier will also decrease and even to 0 . As a result, a large amount of surplus reactive power will be generated at the sending end, resulting in overvoltage correspondingly.

(3) DC current recovery stage: With the removal of the fault, the DC system begins to recover, the DC current and the terminal voltage also begin to return to the steady state value.

\subsection{Mechanism Analysis of Control Parameters of the HVDC System in Suppressing Overvoltage at the Sending End}

According to the analysis of the formation mechanism of transient overvoltage, the main reason for the formation of transient overvoltage is that the DC current decreases to a low value or even 0 due to the trigger angle regulation of the CCA and the VDCOL. So by changing the trigger angle of the rectifier, the overvoltage at the sending end can be suppressed. Trigger Angle of rectifier can be expressed as: 


$$
\alpha=\alpha_{0}+K_{p}\left(I_{d}-I_{\text {ord }}\right)+\frac{1}{T_{i}} \int\left(I_{d}-I_{\text {ord }}\right) d t
$$

where: $\alpha_{0}$ is the initial trigger angle, $I_{\text {ord }}$ is the current setting value, $K_{P}$ and $T_{i}$ are the proportional coefficient and the integral time constant of PI regulator respectively.

$$
I_{\text {ord }}=\left\{\begin{array}{c}
I_{L}, U_{\mathrm{d}}<U_{L} \\
\frac{I_{H}-I_{L}}{U_{H}-U_{L}}\left(U-U_{L}\right)+I_{L}, U_{L}<U_{\mathrm{d}}<U_{H} \\
I_{H}, U_{\mathrm{d}}>U_{H}
\end{array}\right.
$$

where: $I_{L}, I_{H}, U_{L}$ and $U_{H}$ are the minimum current instruction, maximum current instruction, starting voltage and exit voltage of VDCOL link respectively.

It can be seen that the trigger angle of the rectifier can be changed by adjusting the above six parameters reasonably, which influences the voltage at the sending end.

\section{Parameter Optimization Design Scheme}

\subsection{Objective Function}

In this paper, the aim of optimization is to suppress the overvoltage at the sending end, which mainly includes two aspects: the overvoltage amplitude and the overvoltage duration. Therefore, the integral of the difference between overvoltage and steady-state voltage is taken as the objective function in this paper, and the control parameters of the DC system are optimized as the optimization parameters to establish the transient overvoltage optimization model. The objective function $\mathrm{Y}$ can be expressed as:

$$
\begin{aligned}
& S=\int_{t_{1}}^{t_{2}}\left(U_{d g}-U_{d w}\right) d t \\
& Y\left(K_{p}, T_{i}, I_{L}\right)=\min (S)
\end{aligned}
$$

where: $U_{d g}$ is the effective value of overvoltage, $U_{d w}$ is the effective value of steadystate voltage of the system, and $t_{1}$ and $t_{2}$ are the starting and ending time of overvoltage respectively.

\subsection{Genetic Algorithm}

Based on GA, this paper achieves the optimization of control parameters. GA is a search algorithm based on natural selection and population genetic mechanism. The basic process of GA is shown in figure 3. It simulates the phenomena of reproduction, hybridization and mutation in natural selection genetic process. When using GA to solve the problem, the problem of each solution is encoded into a "chromosome", 
namely the individual, several individuals constitute the group (all possible solutions). At the beginning, some individuals are always randomly generated (that is, the initial solution). According to the predetermined objective function, each individual is evaluated and a fitness value is given, the fitness value is used to produce the next generation, choose a few individuals choose operation embodies the principle of survival of the fittest. Good individuals are used for the next generation, while bad individuals will be eliminated.and the individuals chosen by crossover and mutation operator are combined to generate a new generation. A new generation of individuals inherited some excellent characters from last generation, so it's gradually evolving towards the optimal solution [10].

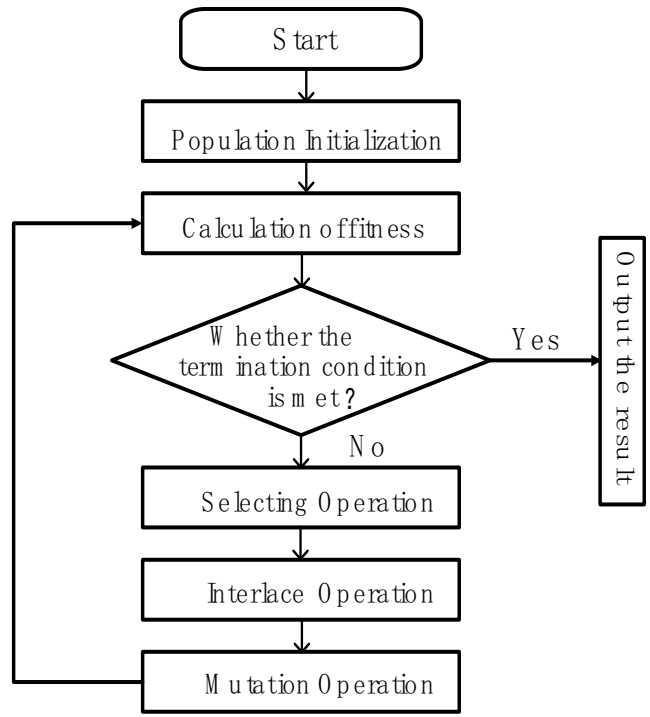

Figure 3. Genetic algorithm schematic diagram.

\subsection{Parameter Optimization Process Based on Genetic Algorithm}

As is shown in figure 4, the specific process of parameter optimization is as follows:

(1) Initialize the GA unit: the initial population number of GA, the number of surviving population, algorithm convergence conditions and the upper and lower limits of optimization parameters.

(2) The HVDC transmission simulation model will start to run the simulation model according to the initialization parameters. After the completion of the first simulation, the simulation model will start to output the voltage parameters at the sending end. The objective function calculation module will start to calculate the objective function value and send the data to Optimum Run module.

(3) The Optimum Run module will call the built-in genetic algorithm after receiving the objective function value data, and the GA unit will output the new parameter optimization value after crossover, mutation and other operations.

(4) The genetic algorithm module judges whether the iteration accuracy meets the requirements through the difference between the two objective function values. If the accuracy does not meet the requirements, the new optimization parameter values are returned to the HVDC simulation model, and the system continues to run in this cycle; 
If the accuracy meets the requirements, the termination signal will be returned to the HVDC transmission simulation model, and the system will terminate operation.

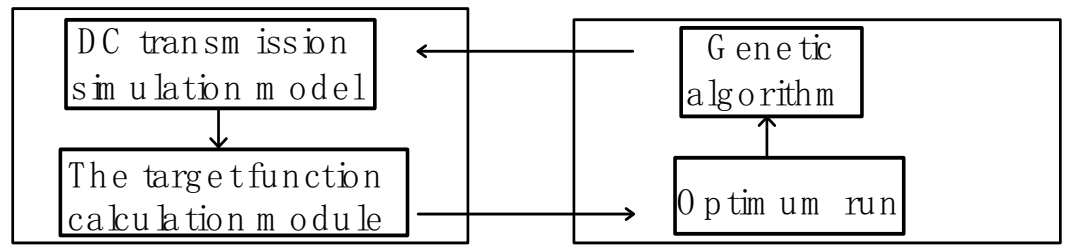

Figure 4. Schematic diagram of parameter optimization operation module.

\section{Case Analysis}

The PSCAD standardized HVDC transmission simulation model is used for simulation verification. The topology of the simulation model is shown in figure 5. Single pole $500 \mathrm{kV}, 1000 \mathrm{MW}$ DC power transmission, there are both 12 pulse converters at rectifying side and inverting side, connected to the weak AC system (frequency: $50 \mathrm{~Hz}$ short circuit ratio: 2.5 ).

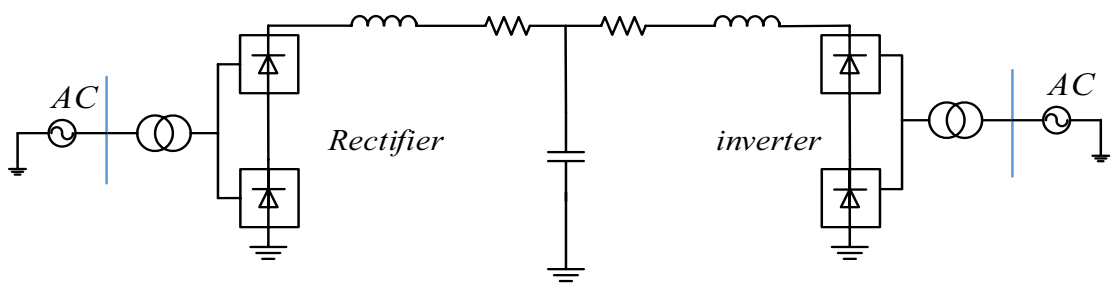

Figure 5. CIGRE_benchmark HVDC transmission simulation system.

Using the model of the HVDC transmission system shown in the figure above, the three-phase short circuit fault of the AC bus at the receiving end was set at $4 \mathrm{~s}$ and the fault time was set as $1 \mathrm{~s}$ to simulate commutation failure at the inverting side. Through many times of optimization, finally the satisfactory parameter value is obtained. The convergence precision is $1 \mathrm{e}-3$, the maximum number of optimization times of the system is 500 , and the simulation time of each optimization is $5 \mathrm{~s}$. The optimal solution of control parameters is finally obtained after multiple optimization. The control parameters of CIGRE HVDC transmission model before and after optimizing by genetic algorithm are listed in table 1.

Table 1. Control parameters before and after the parameter optimization.

\begin{tabular}{llll}
\hline & $\mathbf{I}_{\mathbf{L}}$ & $\mathbf{K}_{\mathbf{P}}$ & $\mathbf{T}_{\mathbf{i}}$ \\
\hline Before optimizing parameters & 0.50 & 0.10 & 0.05 \\
After optimizing parameters & 0.62 & 0.61 & 0.13 \\
\hline
\end{tabular}

Figure 6 compares the voltage waveform at the $\mathrm{AC}$ bus of the sending end before and after parameter optimization respectively. It can be seen that the peak of transient voltage at the sending end reaches $1.28 \mathrm{pu}$ before parameter optimization, and the 
duration of overvoltage is about $200 \mathrm{~ms}$. After parameter optimization, the peak value of overvoltage is $1.03 \mathrm{pu}$, and the duration of overvoltage is about $100 \mathrm{~ms}$. The peak value and duration of overvoltage after parameter optimization are significantly lower than that before parameter optimization, which indicates that the parameter optimization method based on genetic algorithm proposed in this paper is effective. At the same time, the optimized parameters also have a inhibiting effect on the voltage drop by comparing the low voltage peak in the figure. Table 2 compares the peak and duration of overvoltage before and after parameter optimization under four different shortcircuit faults (short-circuit resistance is all $30 \Omega$, short-circuit time is all $0.1 \mathrm{~s}$ ). It can be seen that the parameter optimization scheme designed in this paper is applicable to the commutation failure conditions caused by various short-circuit faults at the receiving end, which has a good suppressing effect on the overvoltage under each fault.

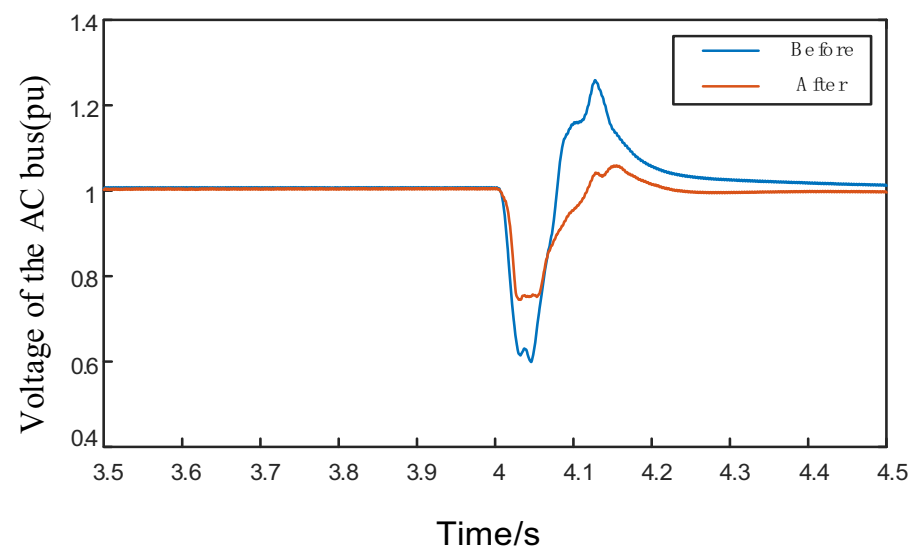

Figure 6. Voltage at feeding side before and after the parameter optimization.

Table 2. Voltage comparison under communication failure caused by different faults.

\begin{tabular}{|c|c|c|c|}
\hline \multirow{2}{*}{ Type of short circuit } & \multicolumn{3}{|c|}{ Overvoltage before parameter optimizationOvervoltage after parameter optimization } \\
\hline & Duration (ms) & Peak (pu) & Duration (ms) \\
\hline Three phase shorting 1.28 & 200 & 1.03 & 60 \\
\hline Single phase earthing 1.25 & 200 & 1.01 & 50 \\
\hline Two phases earthing 1.26 & 120 & 1.02 & 60 \\
\hline Two phases shorting 1.27 & 150 & 1.02 & 65 \\
\hline
\end{tabular}

\section{Conclusion}

Based on the analysis of the mechanism of transient overvoltage at the sending end when commutation failure occurs at the inverting side of the HVDC transmission system, a method of optimizing the DC control parameters based on genetic algorithm to suppress transient overvoltage is proposed in this paper. The following conclusions:

(1) By analyzing mathematical model of HVDC systems, it is concluded that sending the formation of transient overvoltage of is directly related to the reactive power of the converter station, the converter station control system directly determines 
the change of reactive power, therefore, adjusting the parameters of the control system is the most direct inhibition method of transient overvoltage.

(2) Through the analysis of CCA link and VDCOL link, it is concluded that there are six control parameters that affect the reactive power of rectifier station, and by adjusting these six control parameters, the reactive power consumption of converter station can be changed, at the same time the voltage level at the sending end will be affected accordingly. Therefore, a reasonable selection of control parameters can suppress the transient overvoltage at the sending end.

(3) Three main parameters affecting the transient overvoltage are optimized by genetic algorithm. And a set of appropriate control parameters are finally obtained, which can effectively suppress the transient overvoltage at the sending end. The direct relationship between control parameters and transient overvoltage is proved, and the effectiveness of genetic algorithm in HVDC system parameter optimization is also proved.

\section{Acknowledgement}

The paper is funded by the "Offshore Wind Power and Intelligent Energy System Technology Project (Phase I) (HNKJ20-H88)” of Huaneng Group Headquarters Technology Project.

\section{References}

[1] Yang H T, Ji P, Miao M, et al. 2018 Analysis on interrelationship between future UHV power grid structure and power source composition in China Automation of Electric Power Systems 42 (6) 9-17.

[2] Niu Y, Wen J, Wang S and Ma L 2021 Simulation analysis of voltage fluctuation at the sending end of UHVDC system caused by commutation failure 2021 3rd Asia Energy and Electrical Engineering Symposium (AEEES) pp 15-19.

[3] Jin X and Nian H 2021 Overvoltage suppression strategy for sending AC grid with high penetration of wind power in the LCC-HVDC system under commutation failure IEEE Transactions on Power Electronics 36 (9) 10265-10277.

[4] Liu J, Jiang R, Liu Q, Zhang L and Wang R 2018 Study on transient overvoltage of wind farm caused by fault of HVDC system and its suppression measures 2018 2nd IEEE Conf. Energy Int. Energy Syst. Integra. (EI2) pp 1-6.

[5] Luo X, Zhang J, He J, et al. 2015 Coordinated control research of stability control system and pole control system under DC system block considering transient overvoltage Power System Technology 39 (9) 2526-2531.

[6] Mirsaeidi S and Dong X 2020 An enhanced strategy to inhibit commutation failure in line-commutated converters IEEE Transactions on Industrial Electronics 67 (1) 340-349.

[7] Xie G and Wang M 2010 Parameter optimization for current controller in HVDC control system 2010 International Conference on Power System Technology pp 1-5.

[8] Wang F, Liu H, Su G, Liu Y and Fan D 2019 A parameter optimization method for commutation failure prevention in LCC-HVDC system 2019 IEEE 3rd Conference on Energy Internet and Energy System Integration (EI2) pp 1691-1694.

[9] Chen H, Lu H, Wang C, Lin C, Guo Y and Zhang S 2020 Coordinated optimization of HVDC and wind power control parameters for mitigating transient overvoltage on HVDC sending-side system Electric Power Automation Equipment 40 (10) 46-55.

[10] Yu F, Wang X, Yang Y, et al. 2014 The application of a hybrid genetic algorithm in parameter optimization of HVDC constant current controller Power System Protection and Control 42 (9) 126-131. 